\title{
A synthetic approach to terpendoles: decahydrobenzo[f]chromenes by an intermolecular Diels-Alder route
}

\author{
Debjit Basu, ${ }^{a}$ Malapaka Chandrasekharam, ${ }^{* b}$ Prathama S Mainkar, ${ }^{a}$ \\ and Srivari Chandrasekhar*a \\ ${ }^{a}$ Organic Division I, Indian Institute of Chemical Technology, Hyderabad 500 007, India \\ ${ }^{b}$ I\&PC Division, Indian Institute of Chemical Technology, Hyderabad 500 007, India \\ E-mail: srivaric@iict.res.in, chandra@iict.res.in
}

\begin{abstract}
Synthesis of decahydro- $1 H$-benzo[ $f]$ chromene system using intermolecular Diels-Alder reaction has been carried out for the construction of skeleton of terpendole class of terpenoids.
\end{abstract}

Keywords: Indole diterpenoid, intermolecular Diels-Alder reaction, decahydro- $1 H$-benzo[f] chromene system, pyran ring

\section{Introduction}

Indole-diterpenes, isolated from fungi, comprise an important class of architecturally complex and biologically significant metabolites. ${ }^{1}$ These metabolites exhibit biological activities such as tremogenicity, ${ }^{1}$ insecticidal ${ }^{2}$ and pollen growth inhibition. ${ }^{3}$ These natural products share a common core structure comprised of a cyclic diterpene skeleton derived from geranylgeranyl diphosphate (GGPP) and an indole moiety from tryptophan..$^{4-8}$ On the basis of carbon skeleton, it is proposed that these metabolites are biosynthesized by epoxidation of 3-geranyl geranyl indole and subsequent cationic cyclisation similar to the biosynthesis of terpenes and steroids. ${ }^{4,5}$ The isolation and structure elucidation of several of these metabolites are well documented. ${ }^{9}$ Increasing importance of this class of compounds is evident from the potent inhibitory activity of terpendole $\mathrm{C}$ with an $\mathrm{IC}_{50}$ value of $2.1 \mu \mathrm{M}$ followed by that of terpendoles $\mathrm{D}\left(\mathrm{IC}_{50} 3.2 \mu \mathrm{M}\right)$, A $\left(\mathrm{IC}_{50} 15.1 \mu \mathrm{M}\right.$ ), and $\mathrm{B}\left(\mathrm{IC}_{50} 26.8 \mu \mathrm{M}\right)$ (Figure 1) ${ }^{10}$ Recently, a novel action of terpendole $\mathrm{E}$ on motor activity of mitotic kinesin Eg5 has been reported. ${ }^{11}$ The most recent indole-diterpene, JBIR-03, has been isolated from the fungus Dichtomomyces cejpii NBRC 103559 and exhibited anti-MRSA activity and antifungal activity while no reported toxicity towards human cancer cells. ${ }^{12}$ 


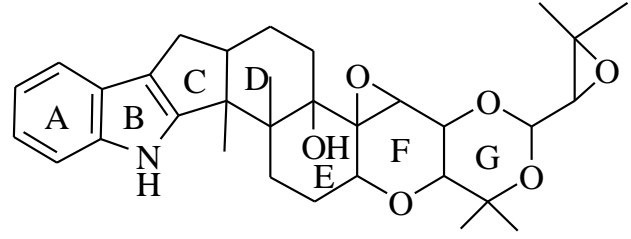

Terpendole A

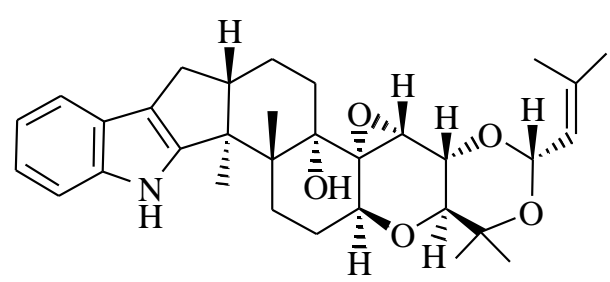

Terpendole $\mathbf{C}$<smiles>CC(C)(O)C1CC2OC23C(CCC2(C)C3CCC3Cc4c([nH]c5ccccc45)C32C)O1</smiles>

Terpendole B

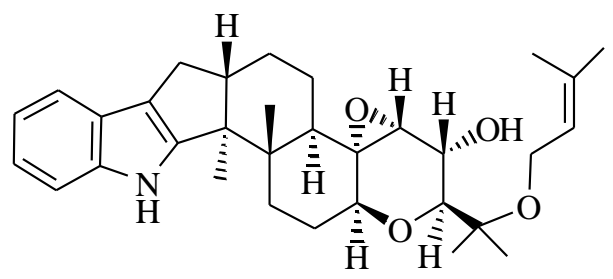

Terpendole D

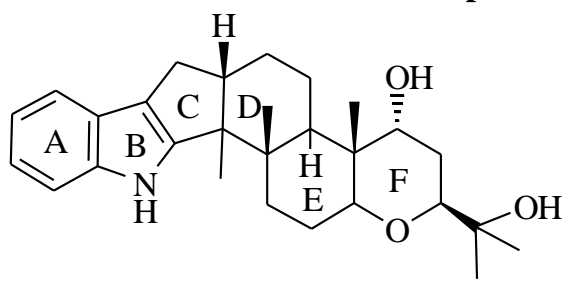

Terpendole E

Figure 1. Structures of representative terpendoles.

Despite their complex molecular structures and potent biological activities, there has not been much focus on their efficient synthesis. ${ }^{13}$ In this paper, we report the construction of key skeleton towards indole-diterpenes. The target framework 1 is envisaged by intermolecular Diels Alder reaction of 4a-methyl-5-vinyl-3,4,4a,7,8,8a-hexahydro- $2 \mathrm{H}$-chromene (2) and (Z)- ethyl-3( $1 H$-indol-2-yl)acrylate (3) (Scheme 1). Commercially available 2-methyl-1,3-cyclohexanedione (4) was selected as starting material for the diene intermediate 2 , as one of the keto functionality could be used as a handle to construct the pyran ring while the other one for diene formation. The dienophile 3 could be obtained from indole-2-carboxylic acid (5).

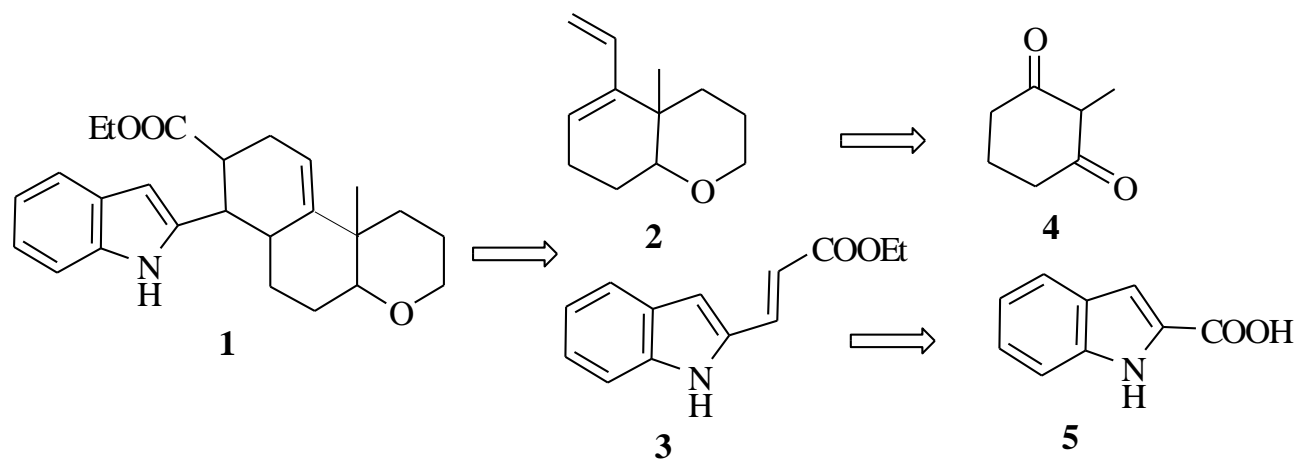

Scheme 1. Retrosynthetic analysis. 


\section{Results and Discussion}

In the first step, 2-methyl-1,3-cyclohexanedione (4) was subjected to alkylation with allyl bromide under standard reaction conditions (potassium carbonate/acetone/reflux) to obtain the desired allyl, methyl dione (6) along with O-alkylated product (6a) in a ratio of 60:40 of the $C$ to $O$-alkylation. ${ }^{14}$ However, use of sodium hydride as a base in dimethylformamide gave exclusively $C$-alkylated product (6) in $89 \%$ yield. Dione (6) was smoothly reduced with sodium borohydride in methanol (93\%) to the corresponding diol (7). Hydroboration of the allylic double bond with borane dimethylsulfide complex gave triol (8) in 91\% yield. Now, the stage is set for the formation of pyran ring by selective tosylation of primary alcohol which acts as a good leaving group, to facilitate in-situ displacement with ring hydroxyl group. The reaction proceeded as expected when the triol (8) was treated with $p$-toluenesulfonyl chloride/triethylamine in dichloromethane to afford the bicyclic pyran (9) (70\%) in one-pot. The ketone handle for diene formation was generated by oxidation of bicyclic carbinol (9) with pyridinium chlorochromate in dichloromethane resulting in the corresponding ketone (10) (87\%). The trans geometry at the ring junction was confirmed by the splitting pattern of the angular proton in ${ }^{1} \mathrm{H}$ NMR spectrum, which displayed large couplings indicating an axial orientation. Vinylation on (10) (vinylmagnesium bromide, tetrahydrofuran, $65 \%$ yield) followed by heating in hexamethylphosphoramide at $210{ }^{\circ} \mathrm{C}$ furnished diene (2) (64\%).

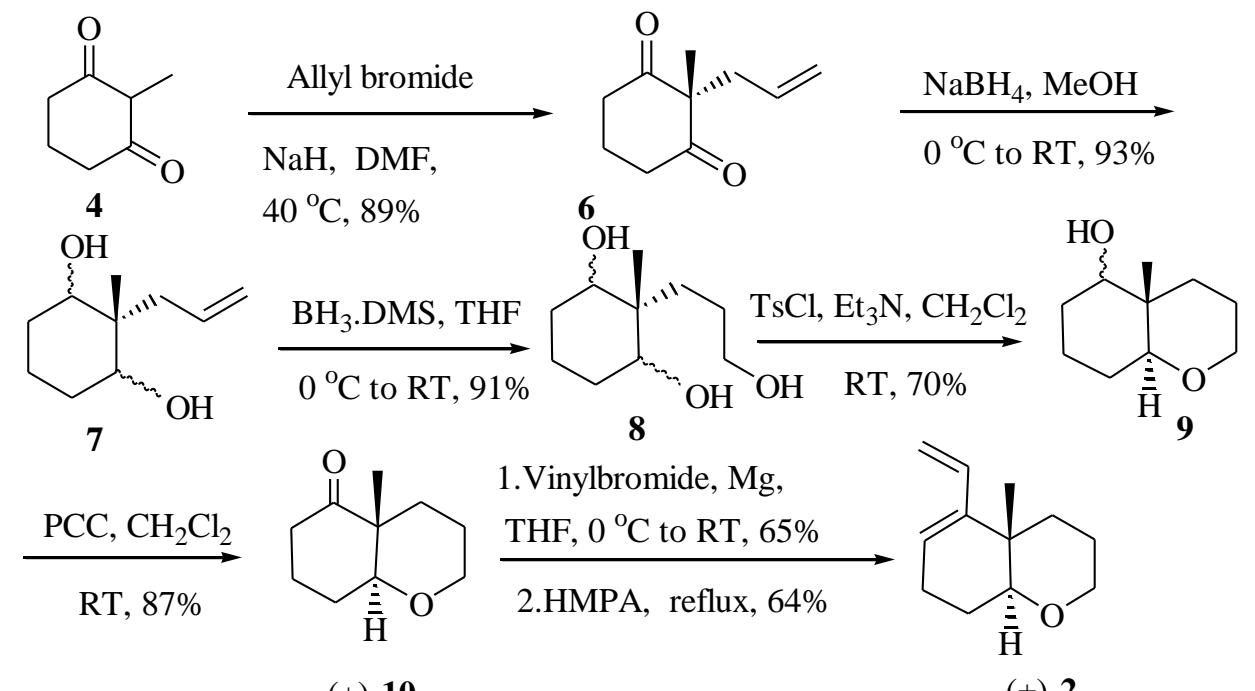

$( \pm)-10$

$( \pm)-2$

Scheme 2. Synthesis of diene 2.

The dienophile (3) was synthesized from indole-2-carboxylic acid (5), which was subjected to esterification with diazomethane to afford the corresponding methyl ester (11) in $97 \%$ yield (Scheme 3). Diisobutylaluminium hydride reduction of the ester at $-78{ }^{\circ} \mathrm{C}$ in dichloromethane produced the corresponding 2 -formylindole (12) in $81 \%$ yield. The aldehyde was subjected to 
Wittig-olefination using (carbethoxymethylene)triphenylphosphorane to obtain ethyl-3-(2indolyl)prop-2-enoate (3) in $95 \%$ yield.

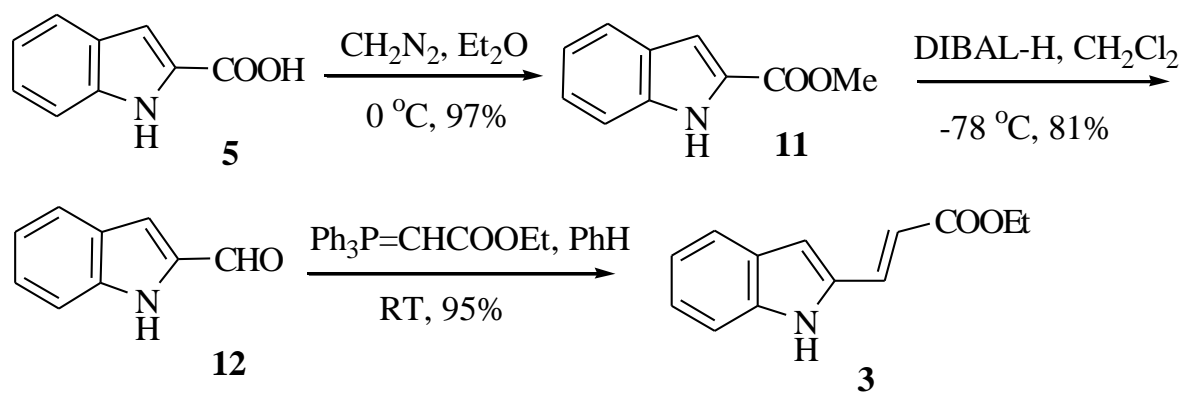

Scheme 3. Synthesis of dienophile.

Having both the diene and the dienophile in hand, the stage is set to carry out Diels-Alder reaction. Diene (2) and dienophile (3), dissolved in dry toluene, were heated in a sealed tube at $110{ }^{\circ} \mathrm{C}$ for 7 days to obtain the desired adduct (1) with $26 \%$ yield. The unreacted starting materials were recovered and reused. Compound (1) was fully characterized as its $N$-Bocderivative (1a) (Scheme 4).

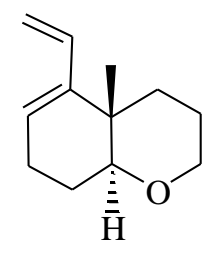

$( \pm)-2$
3, toluene, $110{ }^{\circ} \mathrm{C}$,<smiles>[R]OC(=O)C1CC=C2C(CC[C@H]3OCCC[C@@]23C)C1c1cc2ccccc2[nH]1</smiles>

(Boc) $)_{2} \mathrm{O}, \mathrm{Et}_{3} \mathrm{~N}$,
DMAP, $\mathrm{CH}_{2} \mathrm{Cl}_{2}$,
$\mathrm{RT}, 86 \%$$\quad\left(\begin{array}{l}\mathrm{R}=\mathrm{H} \mathbf{1} \\ \mathrm{R}=\text { Boc 1a }\end{array}\right.$

Scheme 4. Diels-Alder reaction.

\section{Conclusion}

In summary, an advanced complex precursor of indole diterpenoids was accomplished from commercially available starting materials. The use of intermolecular Diels-Alder reaction is significant for the construction of decahydro- $1 H$-benzo[f]chroman framework. 


\section{Experimental Section}

General. All solvents and reagents were purified by standard techniques. Crude products were purified by column chromatography on silica gel of 60-120 mesh. IR spectra were recorded on Perkin-Elmer 683 spectrometer. ${ }^{1} \mathrm{H}$ and ${ }^{13} \mathrm{C}$ NMR spectra were recorded in $\mathrm{CDCl}_{3}$ solution on Brucker Avance 300. Chemical shifts were reported in parts per million with respect to internal TMS. Coupling constants $(J)$ are quoted in Hz. Mass spectra were recorded on CEC-21-11013 or Fannigan Mat 1210 double focusing mass spectrometers operating at a direct inlet system or LC/MSD Trap SL (Agilent Technologies).

2-Allyl-2-methyl-1,3-cyclohexanediol (7). The allyl compound 6 (5.8 g, $34.9 \mathrm{mmol})$ was dissolved in methanol $(50 \mathrm{~mL})$. It was cooled to $0{ }^{\circ} \mathrm{C}$ and then sodium borohydride $(1.32 \mathrm{~g}, 34.9$ $\mathrm{mmol}$ ) was added slowly in portions in the period of $0.25 \mathrm{~h}$. The reaction mixture was stirred at room temperature for $4 \mathrm{~h}$. After completion of the reaction, monitored by TLC, methanol was removed in vacuo. The slurry was cooled to $0{ }^{\circ} \mathrm{C}$ and quenched with saturated sodium chloride and extracted with ethyl acetate $(3 \times 50 \mathrm{~mL})$. The combined organic layer was washed with water $(1 \times 20 \mathrm{~mL})$, brine $(1 \times 20 \mathrm{~mL})$ and then concentrated in vacuo. Residue was purified by silica gel column chromatography (hexane: ethyl acetate 7:3) to obtain the diol 7 as a thick oil. (5.5 g, 93\%). ${ }^{1} \mathrm{H}$ NMR $\left(\mathrm{CDCl}_{3}, 300 \mathrm{MHz}\right): \delta 6.01-5.89\left(\mathrm{~m}, 1 \mathrm{H},-\mathrm{CH}=\mathrm{CH}_{2}\right), 5.16-5.05(\mathrm{~m}, 2 \mathrm{H},-$ $\left.\mathrm{CH}=\mathrm{CH}_{2}\right), 3.73(\mathrm{~m}, 2 \mathrm{H},-\mathrm{CHOH}), 2.29\left(\mathrm{~m}, 2 \mathrm{H}, \mathrm{CH}_{2} \mathrm{CH}=\mathrm{CH}_{2}\right), 1.75-1.23\left(\mathrm{~m}, 6 \mathrm{H},-\mathrm{CH}_{2}-\mathrm{CH}_{2} \mathrm{CH}_{2}-\right.$ ), $0.929\left(3 \mathrm{H}, \mathrm{s},-\mathrm{CH}_{3}\right) .{ }^{13} \mathrm{C} \mathrm{NMR}\left(75 \mathrm{MHz}, \mathrm{CDCl}_{3}\right): \delta 135.4,117.1,73.4,73.0,42.5,40.0,29.6$, 28.5, 18.7, 16.6. ESI (MS): $\mathrm{m} / z 172[\mathrm{M}+\mathrm{H}]^{+}$. IR (KBr): $v 3431,2936,1090 \mathrm{~cm}^{-1}$; Anal. Calcd for $\mathrm{C}_{10} \mathrm{H}_{18} \mathrm{O}_{2}: \mathrm{C}, 70.55 ; \mathrm{H}, 10.66$. Found: $\mathrm{C}, 70.51 ; \mathrm{H}, 10.69$.

2-(3-Hydroxypropyl)-2-methyl-1,3-cyclohexanediol (8). To compound 7 (5.8 g, $34 \mathrm{mmol})$ in anhydrous tetrahydrofuran $(20 \mathrm{~mL})$ was added borane-dimethylsulfide $(3.23 \mathrm{~mL}, 34 \mathrm{mmol}$, $94 \%$ ) over a period of $10 \mathrm{~min}$ maintaining the temperature at $0{ }^{\circ} \mathrm{C}$. Then, the reaction mixture was slowly brought to room temperature, stirred for a period of $8 \mathrm{~h}$ and then treated with $3 \mathrm{~N}$ sodium hydroxide, until the mixture had basic $\mathrm{pH}$ at $0{ }^{\circ} \mathrm{C}$. To this, was added $30 \%$ hydrogen peroxide $(7.7 \mathrm{~mL}, 68 \mathrm{mmol})$ and mixture was stirred for another $3 \mathrm{~h}$.

TLC showed the completion of the reaction. The reaction mixture was diluted with ethyl acetate, washed with water $(1 \times 20 \mathrm{~mL})$ and brine $(1 \times 20 \mathrm{~mL})$. The combined organic layer was concentrated in vacuo and dried over anhydrous sodium sulfate. Residue was purified by column chromatography (hexane: ethyl acetate $2: 8)$ to furnish triol $(8)(5.6 \mathrm{~g}, 91 \%) .{ }^{1} \mathrm{H}$ NMR $\left(\mathrm{CD}_{3} \mathrm{OD}\right.$, $300 \mathrm{MHz}): \delta 5.43\left(\mathrm{~m}, 2 \mathrm{H},-\mathrm{CH}_{2} \mathrm{OH}\right), 5.02(\mathrm{~m}, 2 \mathrm{H},-2 \mathrm{CHOH}), 3.30-2.76(\mathrm{~m}, 12 \mathrm{H}$, all remaining $\mathrm{CH}_{2} \mathrm{~S}$ except $-\mathrm{CH}_{2} \mathrm{OH}$ ), 2.30 (s, 3H, $-\mathrm{CH}_{3}$ ). ${ }^{13} \mathrm{C}$ NMR (75 MHz, $\left.\mathrm{CD}_{3} \mathrm{OD}\right): \delta 74.1,73.7,64.1$, 42.7, 31.8, 30.6, 29.7, 27.0, 19.9, 17.5. ESI (MS): $m / z 189[\mathrm{M}+\mathrm{H}]^{+}$. IR (KBr): v 3403, 2942, $1057 \mathrm{~cm}^{-1}$; Anal. Calcd for $\mathrm{C}_{10} \mathrm{H}_{20} \mathrm{O}_{3}: \mathrm{C}, 63.80 ; \mathrm{H}, 10.71$. Found: $\mathrm{C}, 63.84 ; \mathrm{H}, 10.68$.

4a-Methylperhydrochromen-5-one (9). Triol (8) (5.6 g, $29.78 \mathrm{mmol})$ was dissolved in anhydrous dichloromethane $(50 \mathrm{~mL})$. It was cooled to $0{ }^{\circ} \mathrm{C}$, triethylamine $(8.6 \mathrm{~mL}, 62 \mathrm{mmol})$ and catalytic amount of 4-dimethylaminopyridine $(0.36 \mathrm{~g}, 0.3 \mathrm{mmol})$ was added. The mixture was stirred for another $10 \mathrm{~min}$. $p$-Toluenesulfonyl chloride $(5.6 \mathrm{~g}, 29.78 \mathrm{mmol})$ was added in portions 
at $0{ }^{\circ} \mathrm{C}$. The reaction mixture was stirred overnight at room temperature. After completion of the reaction, monitored by TLC, reaction mixture was diluted with dichloromethane $(20 \mathrm{~mL})$. It was washed with $1 \mathrm{~N}$ hydrochloric acid $(3 \times 20 \mathrm{~mL})$, water $(2 \times 20 \mathrm{~mL})$ and brine $(1 \times 20 \mathrm{~mL})$. The organic layer was dried over anhydrous sodium sulfate and purified by silica gel column chromatography (hexane: ethyl acetate 8:2) to realize the compound (9) as a colourless oil (3.7 $\mathrm{g}$, 70\%). ${ }^{1} \mathrm{H}$ NMR $\left(\mathrm{CDCl}_{3}, 300 \mathrm{MHz}\right): \delta 4.16-3.89\left(\mathrm{~m}, 2 \mathrm{H}, \mathrm{OCH}_{2}\right), 3.52-3.23(\mathrm{~m}, 2 \mathrm{H},-\mathrm{OCH}-$, $\mathrm{OHCH}-), 2.14-0.91\left(\mathrm{~m}, 10 \mathrm{H}, 5 \mathrm{CH}_{2}\right), 0.82\left(\mathrm{~s}, 3 \mathrm{H},-\mathrm{CH}_{3}\right) .{ }^{13} \mathrm{C} \mathrm{NMR}\left(75 \mathrm{MHz}, \mathrm{CDCl}_{3}\right): \delta 82.5$, 68.8, 68.3, 32.5, 30.4, 27.1, 22.2, 19.8, 18.8. ESI (MS): $m / z 171[\mathrm{M}+\mathrm{H}]^{+}$. IR (KBr): v 3427, 2939, 2859, $1079 \mathrm{~cm}^{-1}$; Anal. Calcd for $\mathrm{C}_{10} \mathrm{H}_{18} \mathrm{O}_{2}$ : C, 70.55; H, 10.66. Found: C, 70.57; H, 10.63. 4a-Methylperhydro-5-chromenone (10). Alcohol (9) (3.7 g, $21 \mathrm{mmol})$ and pyridinium chlorochromate $(7.1 \mathrm{~g}, 32 \mathrm{mmol})$ in dry dichloromethane $(50 \mathrm{~mL})$ were stirred under nitrogen atmosphere at room temperature for $4 \mathrm{~h}$. Reaction was monitored by TLC. After completion of the reaction, the mixture was diluted with dichloromethane $(30 \mathrm{~mL})$, washed with brine $(20 \mathrm{~mL})$, water $(20 \mathrm{~mL})$ and dried over anhydrous sodium sulfate. The organic layer was concentrated in vacuo. Crude product was purified by flash column chromatography on silica gel (hexane: ethyl acetate 9:1) to give the keto compound (10) as an oil $(3.2 \mathrm{~g}, 87 \%) .{ }^{1} \mathrm{H} \mathrm{NMR}\left(\mathrm{CDCl}_{3}, 300 \mathrm{MHz}\right)$ : $\delta 3.94$ (m, 1H, OCH-), 3.43-3.16 (m, 1H, $\left.-\mathrm{OCH}_{2}\right), 2.62\left(\mathrm{~m}, 2 \mathrm{H}, \mathrm{COCH}_{2}\right), 2.22-1.38(\mathrm{~m}, 8 \mathrm{H}$, $\left.4 \mathrm{CH}_{2}\right), 1.23\left(\mathrm{~s}, 3 \mathrm{H},-\mathrm{CH}_{3}\right) .{ }^{13} \mathrm{C} \mathrm{NMR}\left(75 \mathrm{MHz}, \mathrm{CDCl}_{3}\right): \delta 209.5,132.0,118.9,64.9,41.0,37.9$, 19.3, 17.3. ESI (MS): $m / z 169[\mathrm{M}+\mathrm{H}]^{+}$. IR (KBr): $v 2949,1707 \mathrm{~cm}^{-1}$; Anal. Calcd for $\mathrm{C}_{10} \mathrm{H}_{16} \mathrm{O}_{2}$ : C, 71.39; H, 9.59. Found: C, 71.41; H, 10.55.

4a-Methyl-5-vinyl-3,4,4a,7,8,8a-hexahydro-2H-chromene (2). Vinyl bromide (37.6 mL, 37.6 mmol, 1M solution) in anhydrous tetrahydrofuran was added to a suspension of magnesium $(0.686 \mathrm{~g}, 28.2 \mathrm{mmol})$ in tetrahydrofuran $(10 \mathrm{~mL})$ at room temperature and stirred for $0.5 \mathrm{~h}$. Then ketone (10) (3.2 g, $18.8 \mathrm{mmol})$ in anhydrous tetrahydrofuran (20 mL) was slowly added to the reaction mixture and it was stirred overnight at room temperature. On completion of the reaction, monitored by TLC, the reaction was quenched with saturated ammonium chloride $(10 \mathrm{~mL})$ and extracted with ethyl acetate $(3 \times 30 \mathrm{~mL})$. The combined organic layer was washed with washed with water $(1 \times 20 \mathrm{~mL})$, brine $(1 \times 20 \mathrm{~mL})$, dried over anhydrous sodium sulfate and evaporated under reduced pressure. The required product was purified by flash column chromatography on silica gel (hexane: ethyl acetate 8:3), to realize the $3^{\circ}$-alcohol $(2.4 \mathrm{~g}, 64.8 \%) .{ }^{1} \mathrm{H} \mathrm{NMR}\left(\mathrm{CDCl}_{3}\right.$, $300 \mathrm{MHz}): \delta 6.36-5.85\left(\mathrm{~m}, 1 \mathrm{H},-\mathrm{CH}=\mathrm{CH}_{2}\right), 5.38-5.06\left(\mathrm{~m}, 2 \mathrm{H},-\mathrm{CH}=\mathrm{CH}_{2}\right), 3.90(\mathrm{~m}, 1 \mathrm{H},-\mathrm{OCH}-)$, 3.60-3.18 (m, 2H, $\left.\mathrm{OCH}_{2}-\right), 2.00-1.09\left(\mathrm{~m}, 10 \mathrm{H}, 5 \mathrm{CH}_{2}\right), 0.98\left(\mathrm{~s}, 3 \mathrm{H},-\mathrm{CH}_{3}\right) .{ }^{13} \mathrm{C} \mathrm{NMR}(75 \mathrm{MHz}$, $\left.\mathrm{CDCl}_{3}\right): \delta 141.7,140.7,113.2,79.6,78.7,68.7,33.5,28.9,27.1,22.5,19.5,14.9$. ESI (MS): $\mathrm{m} / z$ $219[\mathrm{M}+\mathrm{Na}]^{+.} \mathrm{IR}(\mathrm{KBr}): v 3423,2837 \mathrm{~cm}^{-1}$.

The $3^{\mathrm{o}}$-alcohol $(2.4 \mathrm{~g}, 12.2 \mathrm{mmol})$ was dissolved in hexamethylphosphoramide in inert atmosphere. The reaction mixture was heated to $210^{\circ} \mathrm{C}$ for $1 \mathrm{~h}$. TLC showed completion of the reaction. The reaction mixture was diluted with water $(50 \mathrm{~mL})$ and extracted with diethylether $(3$ x $30 \mathrm{~mL})$. The combined organic layer was washed with water $(1 \times 20 \mathrm{~mL})$, brine $(1 \times 20 \mathrm{~mL})$, dried over anhydrous sodium sulfate and evaporated under reduced pressure. The required product 2 was purified by flash chromatography on silica gel (hexane: ethyl acetate 9:1) as an 
oily liquid (1.4 g, 64.2\%). ${ }^{1} \mathrm{H}$ NMR $\left(\mathrm{CDCl}_{3}, 300 \mathrm{MHz}\right): \delta 6.27-6.18\left(\mathrm{~m}, 1 \mathrm{H},-\mathrm{CH}=\mathrm{CH}_{2}\right), 5.63$ $\left(\mathrm{m}, 1 \mathrm{H}, \mathrm{CH}_{2} \mathrm{CH}=\mathrm{C}-\right), 5.25\left(\mathrm{~m}, 1 \mathrm{H},-\mathrm{CH}=\mathrm{CH}_{\mathrm{A}} \mathrm{H}_{\mathrm{B}}\right), 4.94\left(\mathrm{~m}, 1 \mathrm{H},-\mathrm{CH}=\mathrm{CH}_{\mathrm{A}} \mathrm{H}_{\mathrm{B}}\right), 4.00(\mathrm{~m}, 1 \mathrm{H},-$ OCH-), 3.53-3.21 (m, 2H, $\mathrm{OCH}_{2-}$ ), 2.20 (m, 2H, -C=CH- $\left.\mathrm{CH}_{2}\right), 2.01-1.25$ (m, 6H, 3CH $), 1.14$ (s, $\left.3 \mathrm{H},-\mathrm{CH}_{3}\right) .{ }^{13} \mathrm{C} \mathrm{NMR}\left(75 \mathrm{MHz}, \mathrm{CDCl}_{3}\right): \delta 144.4,135.0,121.4,113.7,81.7,69.1,34.0,29.6$, 24.8, 23.7, 22.5, 18.6. ESI (MS): $m / z 201[\mathrm{M}+\mathrm{Na}]^{+}$. IR (KBr): v 3423, $2837 \mathrm{~cm}-1$; Anal. Calcd for $\mathrm{C}_{12} \mathrm{H}_{18} \mathrm{O}: \mathrm{C}, 80.85$; H, 10.18. Found: C, 80.81; H, 10.14 .

t-Butyl-2-(8-(ethoxycarbonyl)-10b-methyl-2,3,4a,5,6,6a,7,8,9,10b-decahydro-1H-benzo[f]chromen-7-yl)-1H-indole-1-carboxylate (1). The diene (2) $(0.20 \mathrm{~g}, 1.12 \mathrm{mmol})$ and dienophile (3) $(0.24 \mathrm{~g}, 1.12 \mathrm{mmol})$ were dissolved in toluene $(2 \mathrm{~mL})$ and heated to $200{ }^{\circ} \mathrm{C}$ in a sealed tube for 8 days. The reaction mixture was cooled to room temperature and diluted with ethyl acetate $(10 \mathrm{~mL})$. It was washed with water $(1 \times 20 \mathrm{~mL})$, brine $(1 \times 20 \mathrm{~mL}))$ and dried over anhydrous sodium sulfate. The crude product was purified by silica gel column chromatography (hexane: ethyl acetate 6:4) and the unreacted diene (2) and dienophile (3) were isolated and recycled. The addition product $1(0.45 \mathrm{~g}$, yield $12 \%)$ was obtained as an oily liquid. Then, (1) was treated with Boc anhydride and triethylamine in dichloromethane to obtain the product (1a) in quantitative yield, which was used for characterization. ${ }^{1} \mathrm{H} \mathrm{NMR}\left(\mathrm{CDCl}_{3}, 300 \mathrm{MHz}\right): \delta 8.23(\mathrm{bs}, 1 \mathrm{H}, \mathrm{NH})$, $7.52(\mathrm{~m}, 1 \mathrm{H}, \mathrm{Ar}-\mathrm{H}), 7.26$ (m, 1H, Ar-H), 7.08 (m, 2H, Ar-H), 6.27 (m, 1H, pyrrole ring Ar-H ), 5.56 (m, 1H, - $\mathrm{CH}=\mathrm{C}-)$, 4.13-3.79 (m, 3H, -OCH-, $\left.\mathrm{COOCH}_{2} \mathrm{CH}_{3}\right), 3.56-3.34\left(\mathrm{~m}, 2 \mathrm{H},-\mathrm{O}_{-} \mathrm{CH}_{2}-\right)$, 3.05-2.31 (m, 5H, - $\mathrm{CHCOOCH}_{2} \mathrm{CH}_{3},-\mathrm{CH}-\mathrm{CH}-\mathrm{COOCH}_{2} \mathrm{CH}_{3},-\mathrm{CH}_{2}-\mathrm{CH}-\mathrm{COOCH}_{2} \mathrm{CH}_{3},=\mathrm{C}-\mathrm{CH}-$ ), 2.14-1.40 (m, 8H, 4CH$), 1.27\left(\mathrm{~s}, 12 \mathrm{H},-\mathrm{NCOOC}\left(\mathrm{CH}_{3}\right)_{3},-\mathrm{CH}_{3}\right), 1.12(\mathrm{t}, J=7.0 \mathrm{~Hz}, 3 \mathrm{H}$, $\left.\mathrm{COOCH}_{2} \mathrm{CH}_{3}\right) .{ }^{13} \mathrm{C} \mathrm{NMR}\left(75 \mathrm{MHz}, \mathrm{CDCl}_{3}\right): \delta 175.3,150.2,146.3,141.5,136.5,128.8,123.50$, 122.6, 119.8, 115.7, 115.6, 107.7, 84.4, 83.6, 68.89, 60.0, 41.1, 40.1, 39.6, 34.9, 34.4, 32.2, 30.7, 28.3, 28.2, 27.9, 27.8, 22.6, 17.0, 14.1. ESI (MS): $\mathrm{m} / z, 516[\mathrm{M}+\mathrm{Na}]^{+}$; Anal. Calcd for $\mathrm{C}_{30} \mathrm{H}_{39} \mathrm{NO}_{5}$ : C, 72.99; H, 7.96. Found: C, 72.95; H, 7.93.

\section{Acknowledgements}

DB thanks CSIR, New Delhi for a research fellowship.

\section{References and Notes}

1. (a) Betina, V. Mycotoxins: Chemical, Biological, and Environmental Aspects; Elsevier: New York, 1989: Vol. 9. (b) Turner, W. B.; Aldridge, D. C. Fungal Metabolites II: Academic Press: London, 1983.

2. Li, C.; Gloer, J. B.; Wicklow, D. T.; Dowd, P. F. Org. Lett. 2002, 4, 3095 and references cited there in.

3. Kimura, Y.; Nishibe, M.; Nakajima, H.; Hamasaki, T.; Shigemitsu, N.; Sugawara, F.; Stout, T. J.; Clardy, J. Tetrahedron Lett. 1992, 33, 6987. 
4. Acklin, W.; Weibel, F.; Arigoni, D. Chimia 1977, 31, 63.

5. De Jesus, E.; Gorst-Allman, P.; Steyn, S.; Van Heerden, R.; Vleggaar, R.; Wessels, L.; Hull, W. E. J. Chem.. Soc., Perkin Trans. 1 1983, 1863.

6. Laws, I.; Mantle, G. J. Gen. Microbiol. 1989, 135, 2679.

7. Byrne, M.; Smith, K.; Ondeyka, G. J. Am. Chem. Soc, 2002, 124, 7055.

8. Munday-Finch, C.; Wilkins, L.; Miles, O. Phytochemistry 1996, 41, 327.

9. (a) Springer J. P.; Clardy J. Tetrahedron Lett. 1980, 21, 231. (b) Cole R. J.; Kirksey J. W.; Wells J. M. Can. J. Microbiol. 1974, 8, 1159. (c) Nozawa K.; Nakajima S.; Kawai K. J. Chem. Soc., Perkin Trans 1 1988, 2607. (d) Gallagher R. T.; Finer J.; Clardy J.; Leutweiler A.; Weibel F.; Acklin W.; Arogoni D. Tetrahedron Lett. 1980, 21, 235. (e) Gallagher R. T.; Clardy J.; Wilson B. J. Tetrahedron Lett. 1980, 21, 239. (f) Cole R. J.; Dorner J. W.; Lamsden J. A.; Cox R. H.; Pape C.; Cunfer B.; Nicolson S. S.; Bedell D. M. J. Agric. Food Chem. 1977, 25, 1197. (g) De Jesus A. E.; Gorstallman C. P.; Steyn P. S.; Vanheerden F. R.; Vleggaa R.; Wessels P. L.; Hull W. E. J. Chem. Soc., Perkin Trans. 1 1983, 1847. (h) Penn J.; Swift R.; Wigley L. J.; Mantlep P. G.; Bilton J. N.; Sheppard R. N. Phytochemistry 1993, 32, 1431. (i) Munday-Finch S. C.; Wilkins A. L.; Miles C. O. J. Agric. Food Chem. 1998, 46, 590. (j) Penn J.; Biddle J. R.; Mantle P. G.; Bilton J. N.; Sheppard R. N. J. Chem. Soc., Perkin Trans. 1 1992, 23. (k) Laakso J. A.; Gloer J. B.; Wicklow D. T.; Dowd P. F. J. Org. Chem. 1992, 57, 2066. (1) Belofsky G. N.; Gloer J. B.; Wicklow D. T.; Dowd P. F. Tetrahedron 1995, 51, 3959. (m) Nakazawa J.; Yajima J.; Usul T.; Ueki M.; Takasuki A.; Imoto M.; Toyoshima Y. Y.; Osada H. Chemistry \& Biology 2003, 10, 131. (n) Miles C. O.; Wilkins A. L.; Garthwaute I.; Ede R. M.; Munday-Finch S. C. J. Org. Chem. 1995, 60, 6067. (o) Ondeyka J. G.; Helms G. L.; Hensens O. D.; Goetz M. A.; Zink D. L.; Tsipouras A.; Shoop W. L.; Slayton L.; Dombrowski A. W.; Polishook J. D.; Ostlind D. A.; Tsou N. N.; Ball R. G.; Singh S. B. J. Am. Chem. Soc. 1997, 119, 8809. (p) Nail J. T.; Mantle P. G.; Sheppard R. N.; Weight E. S. J. Chem. Soc., Perkin Trans. 1 1995, 1121. (q) Li. C.; Gloer J. B.; Wicklow D. T.; Dowd P. F. Org. Lett. 2002, 4, 3095.

10. Huang, X-H.; Tomoda, H.; Nishida, H.; Masuma, R.; Omura, S. J. Antibiot. 1995, 48, 1.

11. Nakazawa, J.; Yajima, J.; Usul, T.; Ueki, M.; Takatsuki, A.; Imoto, M.; Toyoshima, Y. Y.; Osada, H. Chemistry Biology 2003, 10, 131.

12. Ogata, M.; Ueda, J-Y.; Hoshi, M.; Hashimoto, J.; Nakashima, T.; Anzai, K.; Takagi, M.; Kazuo S-Y. J. Antibiot. 2007, 60, 645.

13. (a) Smith, A. B. III, Toshiaki, S., Tamara, L. L., Jill, K.-W.; J. Am. Chem. Soc. 1990, 112, 8197. (b) Smith A. B. III; Haifeng, C., Helv. Chim. Acta 2003, 86, 3908. (c) Smith, A. B. III, Akin H. D., László, K., Org. Lett. 2006, 8, 1665.

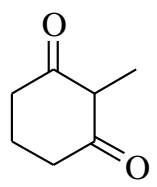

14.
Allyl bromide

Potassium carbonate, Acetone, reflux, 95\%
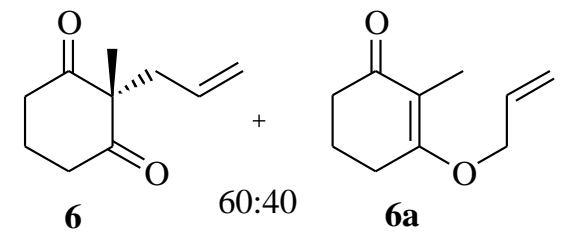

6a 